\title{
Four cases of trisomy 18 syndrome with limb reduction malformations
}

\author{
A L CHRISTIANSON* $\ddagger$ AND M M NELSON $\dagger$ \\ From the Departments of Paediatrics* and Human Genetics $\dagger$, Medical School, University of Cape Town, \\ South Africa.
}

SUMMARY Limb reduction malformations of the arms are well documented in the trisomy 18 syndrome. Four cases of trisomy 18 syndrome with limb reduction malformations of the legs are described and compared with the upper limb malformations.

The first patients with the trisomy 18 syndrome were described by Edwards et $a l^{1}$ and Patau $e t a^{2}$ in 1960 . Since then the clinical features of the syndrome have been characterised, and these have recently been reviewed by Hodes $e t a l^{3}$ and Taylor. ${ }^{4}$

A number of minor abnormalities of the limbs are reported in this syndrome. More severe limb reduction malformations of the arm, especially the forearm, consisting of shortening with hypoplasia and aplasia of preaxial structures, have also been documented in 5 to $10 \%$ of cases..$^{5-9}$ These anomalies are not generally recognised as being associated with the trisomy 18 syndrome. ${ }^{5}$

Severe reduction defects of the legs in infants with trisomy 18 syndrome have been noted in only three published cases. Amputation of one foot was the lesion in two of these patients, ${ }^{510}$ and in the third aplasia of the tibia and hypoplasia of the first metatarsal were described. ${ }^{5}$ The purpose of this paper is to present four cases of trisomy 18 syndrome and to draw attention to the association with gross limb reduction malformations mainly in the lower limbs.

\section{Case reports}

Our patients were among a group of 50 infants with trisomy 18 syndrome, referred to the Department of Human Genetics, University of Cape Town, between 1971 and 1983. Chromosomal studies confirmed the diagnosis in all instances. The clinical and radiological data were obtained from the departmental and hospital records.

\section{PATIENT 1}

A black female was delivered at 37 weeks' gestation by Caesarean section, because of persistent breech

$\ddagger$ Present address: Chelmsford Medical Centre, 107 Chelmsford Road, Durban, Natal, South Africa.

Received for publication 5 December 1983.

Accepted for publication 10 January 1984. position, to a 43 year old mother who had six normal children from previous pregnancies. Physical examination revealed a small female infant with all growth parameters less than the 3rd centile for gestational age. She had numerous dysmorphic features typical of the trisomy 18 syndrome. These included hypotonia, microcephaly, a prominent occiput and glabella, low set malformed ears, apparent hypertelorism, a high arched palate, and micrognathia. The heart was clinically enlarged and a systolic murmur was heard over the praecordium. Examination of the abdomen and respiratory system yielded no abnormalities, but the anus was ectopically situated and the clitoris enlarged.

The arms were flexed at the elbow and the hands were clenched with the index finger overlapping the third finger and the fifth finger over the fourth. Bilateral simian creases and hypoplastic nails were noted. The most striking features were the legs, which were short and malaligned at knee and ankle. The left foot had six digits with almost complete syndactyly between the first and second toes, but only partial fusion of the third, fourth, fifth, and sixth digits. The right foot had four digits (fig 1).

Radiographs of the pelvis and lower limbs showed dislocation of the left hip and a single long thin bone in both lower legs. A calcaneus was present in both feet (fig 1). The first metatarsal of the left foot was hypoplastic while the remaining four were moderately well formed. The phalanges of the syndactylous preaxial first two digits appeared normal but those of the remaining toes were hypoplastic. The right foot had only four metatarsals, that of the big toe being missing. The phalanges were hypoplastic.

PATIENT 2

A black female was delivered normally at term to a 
43 year old mother who had six normal children from previous pregnancies. Physical examination revealed a small female infant, with all growth parameters below the third centile, reduction malformations of the legs, a lumbosacral meningomyelocele, and several dysmorphic features typical of the trisomy 18 syndrome. A cardiac murmur compatible with a small ventricular septal defect was heard. The respiratory system, abdomen, and genitalia were normal, but the anus was patulous.

Both arms were flexed at the elbow and markedly hypertonic. The right hand exhibited preaxial polydactyly with syndactylous thumbs and a hypoplastic index finger. The legs were flaccid and short, with popliteal webs resulting in fixed flexion deformities at the knees. The left knee could be dislocated. Bilateral talipes calcaneovarus was present with complete torsion of the feet. The right foot had preaxial oligodactyly with three digits. The left foot had four digits owing to syndactyly between the second and third toes (fig 2).

Radiographs of the pelvis and legs revealed

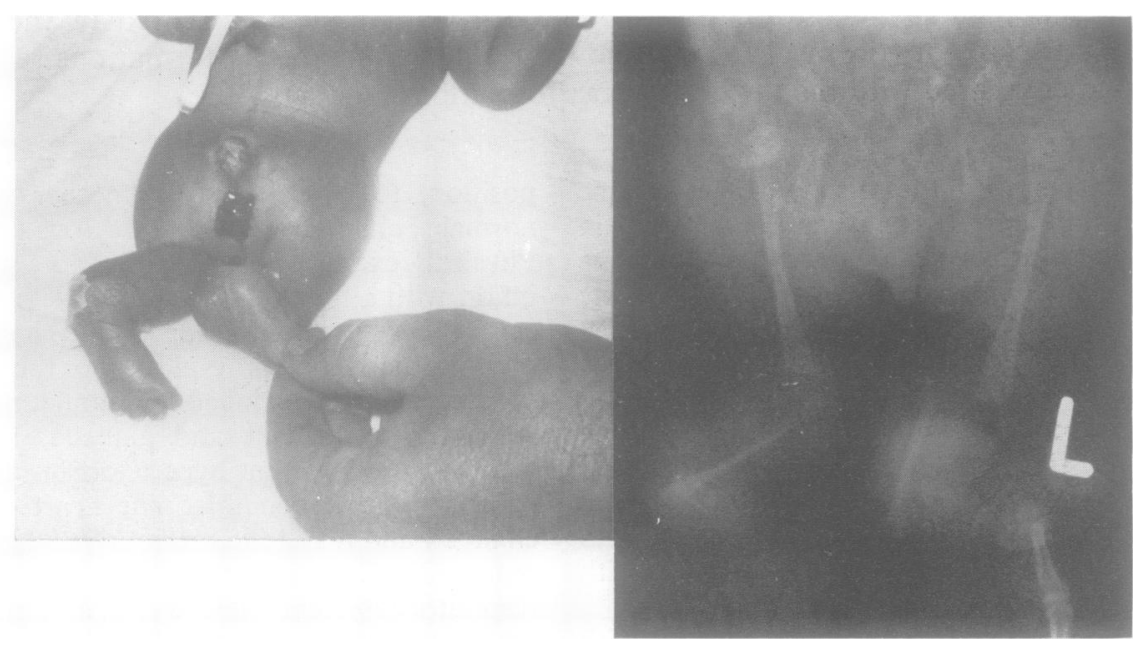

II I Patient 1: clinical pliotograph and radiograph of lower limbs.

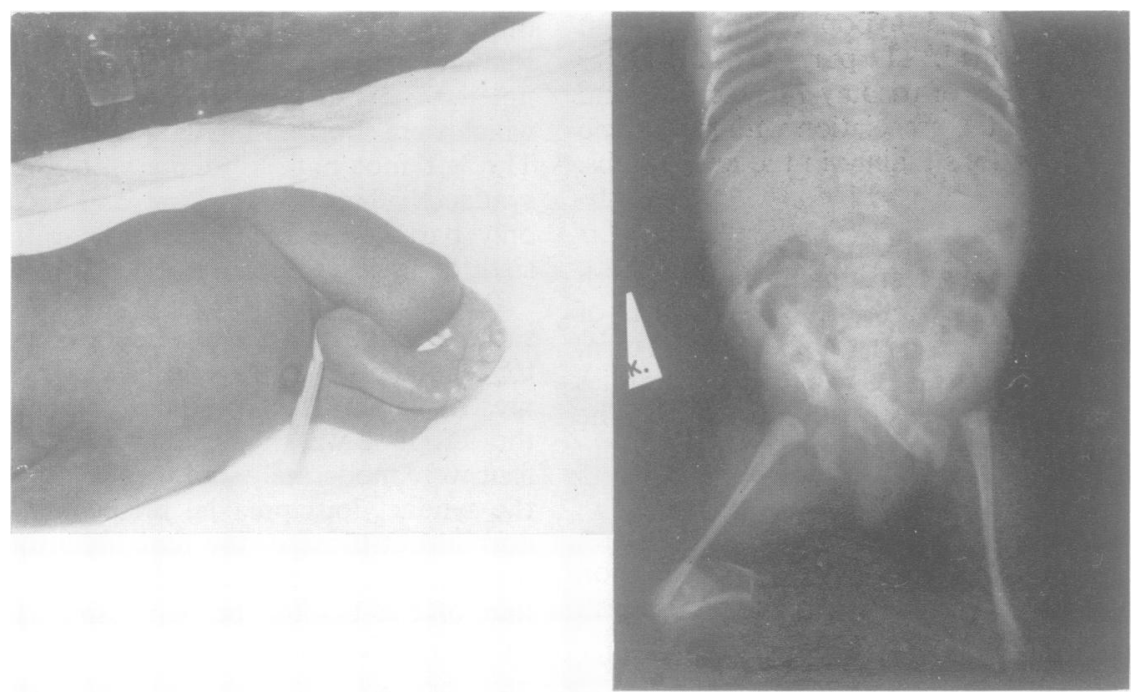

Haci 2 Patient 2: clinical photograph and radiograph of loner limbs. 
bilateral dislocation of the hips and a single long bone in both lower legs. The right foot had a calcaneus, talus, and four hypoplastic metatarsals. The talus was absent in the left foot and there were five hypoplastic metatarsals. Only three phalanges were visible in the radiograph of the right foot. In the left foot the five proximal phalanges appeared normal but that of the first toe was at a $45^{\circ}$ angle to the metatarsal (fig 2).

PATIENT 3

A black male was delivered as a breech at 36 weeks'

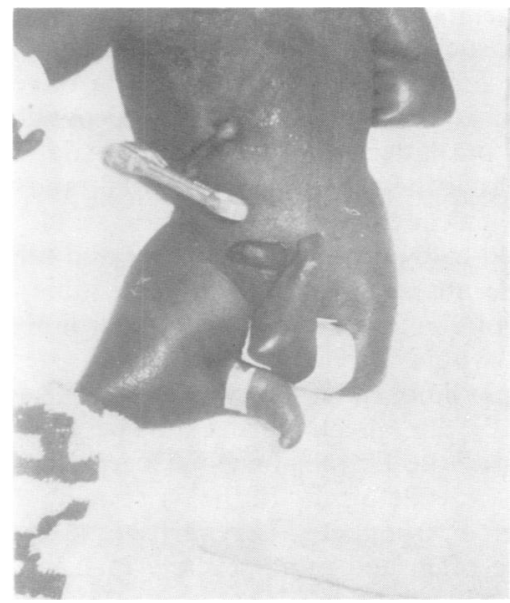

HIG 3 Patient 3: clinical photograph. gestation to a 36 year old mother with three normal children from previous pregnancies. Physical examination revealed a small cyanosed infant, with all growth parameters below the 3rd centile for gestational age, and reduction malformations of both legs and the left arm. He had numerous dysmorphic features typical of the trisomy 18 syndrome. A systolic cardiac murmur was heard over the praecordium. Examination of the abdomen and respiratory system was normal. The scrotum was flat and neither testis could be palpated; the penis appeared normal.

The right arm was flexed at the elbow and a varus deformity of the left wrist was noted. The left thumb was missing and the nails of the remaining four fingers were hypoplastic. The intact right hand was flexed at the wrist and the fingers were in fixed flexion. The lower limbs were shortened, owing in part to dislocation of the hips. Popliteal webs resulted in flexion deformities of both knees, and the left knee could be dislocated. The left foot had two stubs of digits while the right foot had monodactyly; both showed severe talipes calcaneovarus (fig 3).

Radiographs of the pelvis and lower legs confirmed dislocation of the hips. The left femur was thin and hypoplastic and only a single long bone was demonstrable in both lower legs. The calcaneus was present in both feet, together with a single hypoplastic metatarsal bone in the right foot and two similar structures in the left foot. Radiographs of the left arm showed absence of the radius, four metacarpal bones, and a varus deformity of the wrist.

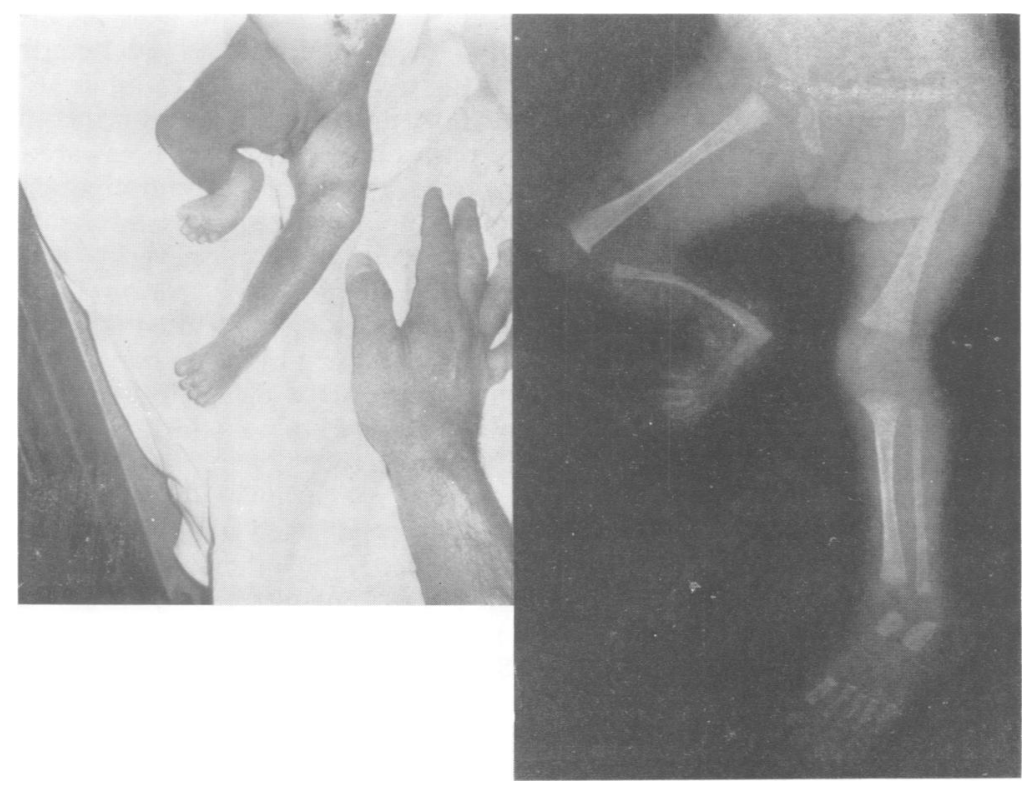

FIG 4 Patient 4: clinical photograph and radiograph of lower limbs. 
PATIENT 4

A female of mixed ancestry was delivered normally at term. Her mother, aged 42 years, had six normal children from previous pregnancies. Physical examination revealed a small infant with a limb reduction malformation of the right leg and numerous dysmorphic features. These included microcephaly, a flattened occiput, low set malformed ears, a right sided auricular tag, microphthalmia of the left eye, micrognathia, webbing of the neck associated with redundant skin folds, and a cleft lip and palate. A systolic cardiac murmur compatible with a ventricular septal defect was present. The respiratory system and abdomen were normal, but the clitoris was hypertrophied and bifid.

Postaxial polydactyly on the left hand and syndactyly between the second and third digit on the right hand were noted. The right leg was shortened, owing in part to dislocation of the right hip and the reduction malformation of the lower leg. It was abducted at the hip, flexed at the knee due to a popliteal web, and a talipes calcaneovarus deformity was present. The left foot had a rocker bottom shape with a shortened hallux and hypoplastic toenails (fig 4).

Radiographs of the lower limbs and pelvis demonstrated dislocation of the left hip and a single, fractured long bone in the right lower leg. Hypoplasia of the metatarsals of both feet was noted, but the first metatarsal of the right foot was smaller and more hypoplastic than the other (fig 4).

\section{Discussion}

It is difficult to explain the paucity of reports of cases with severe leg reduction malformations in trisomy 18 syndrome. Pfeiffer and Sautelmann ${ }^{5}$ commented that "minor anomalies of the thumb may be overlooked and major malformations such as pseudophocomelia have a greater chance of being published", a statement made with reference to the upper limbs. The lower limb defects in our patients were striking and could not have been omitted from previous major reviews.

The parental age distribution, age at death of the infants, and season of birth of these four cases show no marked differences from the series reported in the Caucasian races of the northern hemisphere nor from the other cases of trisomy 18 syndrome studied in our cytogenetic laboratory.

Several features were noted in common in the legs of our patients. In each case only the distal leg was reduced in length and this was bilateral in three instances. Gross talipes calcaneovarus was seen in all affected limbs. Preaxial polydactyly or oligodactyly was present in three patients and the hallux of the affected limb in the fourth patient was markedly shortened. Other shared findings were dislocatable knees, popliteal webs, and dislocation of the hips.

Radiographs were similar in all four patients. Because of the severe malformations, it was difficult to obtain perfect $x$-rays, but only one hypoplastic long bone was seen in the lower leg of the affected limbs. The calcaneus was present in all cases, but only in patient 2 could a talus be seen. Hypoplastic metatarsals were noted in all the feet. In patients 1 and 2 the preaxial oligodactyly was associated with aplasia of the first metatarsal and in patient 4 the first metatarsal of the affected foot was more hypoplastic than the others. It is a matter of conjecture as to whether the single long bone in these affected lower limbs was a tibia or fibula. We favour the latter bone as other preaxial structures were also either absent or abnormal, and the gross talipes calcaneovarus deformities would be predisposed to by the absence of tibia and talus.

If the absent bone is indeed the tibia, then the reduction malformations of the lower limbs of these infants would appear to parallel those described in the upper limbs of infants with trisomy 18 syndrome. Clinically, the affected arms showed hypoplasia or aplasia of the thumb, first metacarpal, and radius, with a varus deformity of the wrist being a common feature. ${ }^{56}$ Asymmetry is reported and the left arm is more often and more severely involved than the right, as in our case 3 .

There are two reasons for drawing attention to these limb abnormalities. Firstly, antenatal ultrasound examination is now routine in many centres and the detection of such defects as we describe should occur, as the overall incidence is about $16 \%$ in the syndrome $(8 \%$ in the upper limb and $8 \%$ in the lower). The ultrasonologist should be aware of the association with abnormal chromosome patterns, and amniotic fluid cell chromosome studies may then be initiated. Secondly, the severity of the limb problems noted by the paediatrician in the neonatal examination may distract attention from the other relatively minor physical changes and chromosome investigations be delayed, as actually happened in our case 4 who was the first of the series and born 8 years ago.

Counselling is often sought on the risk of recurrence in sibs of children affected with limb defects such as described here. If no other abnormality is present, the risk is low, but ultrasound scans may be offered for reassurance. If a chromosome defect like trisomy 18 is present, then the recurrence risk is probably equal to the age associated risk of chromosomal defect. This age associated risk has been calculated for Down's syndrome to be 
approximately 1 in 3000 for mothers of 30 years or under, rising to 1 in $\mathbf{4 0}$ for mothers of 40 and over. It has not been estimated for trisomy 18 by itself, but an extra chromosome 18 is the second most common anomaly found in antenatal chromosome studies. Amniocentesis should be offered in subsequent pregnancies to the mothers of babies with trisomy 18 such as we have presented. This, with ultrasound examination, will allow reassurance or detection of abnormality.

We would like to thank the different physicians who referred these infants to the Cytogenetic Laboratory and who have allowed us access to the available data. The secretarial assistance of Mrs P Smart and Miss G Abrahams is gratefully acknowledged.

\section{References}

1 Edwards JH, Harnden DG, Cameron AH, et al. A new trisomic syndrome. Lancet $1960 ; \mathbf{i}: 787-90$.

2 Patau K, Smith DW, Therman E, et al. Multiple congenital anomaly caused by an extra autosome. Lancet $1960 ; \mathbf{i}: 790-3$.

${ }^{3}$ Hodes ME, Cole J, Palmer CG, Reed T. Clinical ex- perience with trisomies 18 and 13. J Med Genet 1978;15: 48-60.

4 Taylor AI. Autosomal trisomy syndromes: a detailed study of 27 cases of Edwards' syndrome and 27 cases of Patau's syndrome. J Med Genet 1968;5:227-52.

5 Pfeiffer RA, Sautelmann R. Limb anomalies in chromosomal aberrations. Birth Defects 1977;13:319-37.

6 Bofinger MK, Dignan PS, Schmidt RE, et al. Reduction malformations and chromosome anomalies. Am J Dis Child $1973 ; 125: 135-43$.

7 Voorhess ML, Aspillaga MJ, Gardner LI. Trisomy 18 syndrome with absent radius, varus deformity of hand and rudimentary thumb. J Pediatr 1964;65:130-3.

8 Rabinowitz JG, Moseley JE, Mitty HA, et al. Trisomy 18 , esophageal atresia, anomalies of the radius and congenital hypoplastic thrombocytopenia. Radiologv 1967;89:488-91.

9 Kajii T, Kiyoshi O, Katsuaki I, et al. A probable 17-18 trisomy syndrome with phocomelia, exomphalos, and agenesis of hemidiaphragm. Arch Dis Child 1964;39: 519-22.

10 Werchsel ME, Luzzatti L. Trisomy 17-18 syndrome with congenital extrahepatic bilary atresia and congenital amputation of the left foot. J Pediatr 1965;67:324-7.

Correspondence and requests for reprints to Dr M M Nelson, Department of Human Genetics, Medical School, University of Cape Town, Observatory 7925, South Africa. 